\title{
Sliding Mode Control of Underactuated Nonlinear Systems Based on Piecewise Double Power Reaching Law
}

\author{
Congcong Yue $\mathbb{D}^{1,2}$ Haisheng $Y u(\mathbb{D})^{1,2}$ and Xiangxiang Meng $\mathbb{D}^{1,2}$ \\ ${ }^{1}$ College of Automation, Qingdao University, Qingdao, Shandong, China \\ ${ }^{2}$ Shandong Province Key Laboratory of Industrial Control Technology, Qingdao University, Qingdao 266071, Shandong, China
}

Correspondence should be addressed to Haisheng Yu; yu.hs@163.com

Received 31 October 2021; Revised 6 January 2022; Accepted 18 January 2022; Published 11 February 2022

Academic Editor: Javier Moreno-Valenzuela

Copyright $(92022$ Congcong Yue et al. This is an open access article distributed under the Creative Commons Attribution License, which permits unrestricted use, distribution, and reproduction in any medium, provided the original work is properly cited.

A sliding mode control (SMC) strategy using novel piecewise double power reaching law (PDPRL) for a class of fourth-order nonlinear underactuated systems (USs) is proposed to realize simultaneous control of the unactuated and the actuated part. A novel PDPRL is designed in the form of a piecewise function to reduce the chattering produced by SMC. The fixed-time convergence characteristic and the existence of maximum convergence time independent of the initial value of the sliding mode surface for the PDPRL are analyzed. Considering the uncertainties of model parameters and external disturbance in the USs, a supertwisting disturbance observer (STDO) is provided to accurately estimate the disturbance in real time, and the estimated value is compensated to the controller. The underactuated inverted pendulum system is taken to verify the effectiveness of the devised strategy, and the simulation results show that the proposed strategy can reduce chattering, suppress the disturbance, and enhance the robustness of the system.

\section{Introduction}

Underactuated systems (USs) are the systems in which the spatial dimension of the control input vector is less than the configuration dimension of the system, i.e., the control inputs are less than the degrees of freedom of the system [1-3]. USs require fewer drivers and can be built with some of the drivers-related equipment removed in fully-actuated systems, which have very important significance in reducing volume, weight, energy consumption, and cost of the system. Therefore, they are widely used in mobile robots [4], manipulators [5], WAcrobot [6], underwater vehicles [7], and other control fields. Because of the wide application of USs in practice and theoretical obstacles, the demand for these systems has grown dramatically, and the demand for control precision for USs is more and more accurate due to the complexity of conditions in the practical application of these fields. However, the research of the stabilization and tracking control of USs has been studied by many scholars in the field of control in recent decades [8-11]. The lack of actuators in USs still brings a lot of difficulties to design the controllers; the control problem has always been the research subject that the experts study diligently. Therefore, the research on USs has important theoretical and practical significance.

Sliding mode control (SMC) [12-14] which has the characteristics of independent of model parameters, easy implementation, and strong robustness is widely used in nonlinear systems. In practical applications, external disturbances and model parameter uncertainty will affect the control accuracy of the US and even cause system instability. At this time, only relying on SMC cannot meet the practical requirement. Thus, there are control method based on disturbance observer, adaptive SMC method, improved second-order SMC method [15], etc. The SMC method based on disturbance observer can enhance the robustness of the system by estimating the disturbance and compensating the estimated value to the controller [16, 17]. The adaptive method can solve the problem of model parameters uncertainty by designing adaptive law and eliminate the influence on the system $[18,19]$. But, for the general form of the state equation of the USs without any form of transition, the traditional SMC method can only control the unactuated or actuated part of the USs, but not both. In [20], a decoupled SMC method was proposed to realize the 
simultaneous control of unactuated and actuated parts of the USs. The authors of [21] further designed a decoupled nonsingular terminal SMC (DTSMC) strategy to improve the dynamic response of the system and solve the singularity problem of terminal SMC. In order to improve the convergence performance of the system, a fast nonsingular terminal SMC method was proposed in [22]. However, SMC can cause chattering of the system which will cause the system to consume unnecessary energy and affect the control performance of the system. Therefore, chattering is still an important problem to be solved.

For the chattering problem caused by SMC, a large number of effective studies have been done by a lot of experts; the boundary layer method [23], fast terminal SMC $[24,25]$, high-order SMC [26, 27], the control method based on reaching law $[28,29]$, and so on are presented. The boundary layer function was adopted in [20], the saturation function in the boundary layer function was adopted instead of the sign function, and the system states were kept on sliding mode surfaces by setting the boundary coefficient to reduce the chattering. Although it could effectively reduce the chattering, the control precision of the controller would be greatly affected. The high-order SMC method could effectively reduce the chattering, but the selection of the sliding mode surface depended on the system states and the time derivative of the controller input, which were hard to obtain. In addition, this method was difficult to apply to first-order systems [30]. Terminal SMC could effectively suppress chattering and reach the steady state of the system, but it could not eliminate the chattering and the convergence speed was slower than that of SMC with exponential reaching law $[31,32]$. The terminal SMC method was similar to the SMC method which adopts the reaching law in terms of mathematical structure, except that the former was concerned with the movement of the system on the sliding mode surfaces and the latter was concerned with the motion of the reaching stage [33]. Gao analyzed the causes of chattering from various aspects and established exponential reaching law, the power reaching law, constant reaching law, and so on [34]. These reaching laws lay an important foundation for the emergence of other reaching laws, and various reaching laws for continuous and discrete-time systems have recently been adopted $[35,36]$. Although the power reaching law could theoretically eliminate chattering, when the distance between the sliding mode surfaces and the system states is large, the approaching rate is small. In [37], a double power reaching law (DPRL) was described; compared with fast power reaching law (FPRL), it exhibited a faster approaching speed even if the system was farther from the sliding mode surface and a lower approaching rate as the system near the surface; also, smooth dynamics was realized on the basis of weakening chattering. In [29], based on the exponential reaching law, a reaching law including the system state variable and the sliding surface as a power term was designed.

Therefore, on the basis of literature [37], a novel piecewise DPRL (PDPRL) is proposed to reduce chattering and increase the reaching speed of the system. The PDPRL which combines the advantages of piecewise function and
DPRL is used to separate the reaching process of the system arrive at the sliding mode surface into two stages, $|s| \geq 1$ and $|s|<1$, with 1 as the demarcation point; then, the system can have a large approaching speed no matter which stage it is in. The characteristics of convergence in fixed time for proposed PDPRL are analyzed, which shows that the reaching law has maximum convergence time and the time is not concerned with the initial value of the sliding mode variable. Meanwhile, considering the uncertainties of model parameters and external disturbance on the system, a super twisting disturbance observer (STDO) is adopted to estimate the lump disturbance; also, the estimated value is compensated to the controller to eliminate negative effects. The devised SMC strategy using novel PDPRL and STDO is applied to the underactuated inverted pendulum system, and simulation is carried out by comparing with the existing control strategies.

\section{System Description and Control Strategy}

In this part, the system model is briefly described, and a control strategy to ensure the stabilization of the whole system with and without the lump disturbance is proposed. The stability of the system using the devised strategy is also analyzed.

The dynamic model of the fourth-order nonlinear underactuated systems is constructed as [20]

$$
\begin{aligned}
& \dot{x}_{1}=x_{2}, \\
& \dot{x}_{2}=f_{1}(\mathbf{x})+b_{1}(\mathbf{x}) u, \\
& \dot{x}_{3}=x_{4}, \\
& \dot{x}_{4}=f_{2}(\mathbf{x})+b_{2}(\mathbf{x}) u,
\end{aligned}
$$

where $u$ is the control input, $\mathbf{x}=\left[\begin{array}{llll}x_{1} & x_{2} & x_{3} & x_{4}\end{array}\right]^{\mathrm{T}}$ is the state vector, and $f_{1}(\mathbf{x}), f_{2}(\mathbf{x}), b_{1}(\mathbf{x}) \neq 0$, and $b_{2}(\mathbf{x}) \neq 0$ are nonlinear functions that represent the dynamics of the system. The system described in equations (1)-(4) is a general underactuated system, which appears in inverted pendulum system, tower crane system, Pendubot system, ball-beam system, and other systems. The aim of control is to make the system reach the desired position

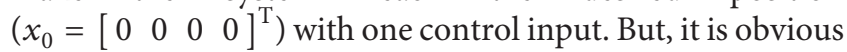
that the control input $u$ can only achieve the control of the states $x_{1}$ and $x_{2}$ or $x_{3}$ and $x_{4}$, not both. As a result, a special decoupled method is adopted in Section 2.1 to deal with the coupling problem, and the unactuated and actuated parts of the system can be controlled through a control input.

\subsection{PDPRL-DTSMC without Disturbance}

2.1.1. Decoupled Terminal Sliding Mode Control. For the first time, the decoupled SMC method is designed in 1998 to find a solution to the natural coupling problem of the nonlinear systems with fourth-order. The contribution of the decoupled method is to decouple a class of nonlinear systems similar to systems (1)-(4) into two subsystems A and B with 
second-order; subsystem A contains states $x_{1}$ and $x_{2}$; subsystem $\mathrm{B}$ contains states $x_{3}$ and $x_{4}$. Thus, realizing the decoupled of states $x_{1}, x_{2}$ and $x_{3}, x_{4}$, subsystem $\mathrm{A}$ and subsystem $B$ are regarded as the primary target and the secondary target (it depends on the question as to which subsystem to choose as the primary or secondary target); $s_{1}$ and $s_{2}$ are the nonsingular terminal sliding mode surfaces of them, respectively. Because the control of these two goals is achieved by controlling the primary target, the information of the secondary target must reflect the primary target by an intermediate signal $z$; then, the sliding surface $s_{2}=0$ of subsystem $B$ is embedded to the primary target and realized DTSMC.

Now, with $s_{1}=0$ and $s_{2}=0$, the control goal is to achieve the convergence of subsystems $\mathrm{A}$ and $\mathrm{B}$ to the desired equilibrium points $\left[\begin{array}{ll}x_{10} & x_{20}\end{array}\right]^{\mathrm{T}}=\left[\begin{array}{ll}0 & 0\end{array}\right]^{\mathrm{T}}$ and $\left[\begin{array}{ll}x_{30} & x_{40}\end{array}\right]^{\mathrm{T}}=\left[\begin{array}{ll}0 & 0\end{array}\right]^{\mathrm{T}}$ by one control input, respectively.

Here, $s_{1}$ and $s_{2}$ are chosen as

$$
\begin{aligned}
& s_{1}=\lambda_{1}\left|x_{1}-z\right|^{\gamma_{1}} \operatorname{sign}\left(x_{1}-z\right)+x_{2}, \\
& s_{2}=\lambda_{2}\left|x_{3}\right|^{\gamma_{2}} \operatorname{sign}\left(x_{3}\right)+x_{4},
\end{aligned}
$$

where $\lambda_{1}$ and $\lambda_{2}$ are positive parameters, $1<\gamma_{1}<2,1<\gamma_{2}<2$. Intermediate signal $z$ is selected as

$$
z=p \operatorname{sat}\left(\frac{s_{2}}{\phi_{s}}\right)
$$

where $p(0<p<1)$ is a constant, $\phi_{s}$ is the boundary layer of $s_{2}$, and sat $\left(s_{2} / \phi_{s}\right)$ is saturation function and expressed as

$$
\operatorname{sat}\left(\frac{s_{2}}{\phi_{s}}\right)= \begin{cases}\operatorname{sign}\left(\frac{s_{2}}{\phi_{s}}\right), & \left|\frac{s_{2}}{\phi_{s}}\right| \geq 1, \\ \frac{s_{2}}{\phi_{s}}, & \left|\frac{s_{2}}{\phi_{s}}\right|<1 .\end{cases}
$$

Now, as $s_{1}=0$ and $s_{2}=0$, the previous control objective $\left[\begin{array}{ll}x_{10} & x_{20}\end{array}\right]^{\mathrm{T}}=\left[\begin{array}{ll}0 & 0\end{array}\right]^{\mathrm{T}}$ of subsystem $\mathrm{A}$ has been changed to $\left[\begin{array}{ll}x_{10} & x_{20}\end{array}\right]^{\mathrm{T}}=\left[\begin{array}{ll}z & 0\end{array}\right]^{\mathrm{T}} \mathrm{T}$, and that of subsystem $\mathrm{B}$ is still $\left[\begin{array}{ll}x_{30} & x_{40}\end{array}\right]^{\mathrm{T}}=\left[\begin{array}{ll}0 & 0\end{array}\right]^{\mathrm{T}}$. As can be seen from (7), $z$ is an attenuating oscillation signal that approaches zero and a function of $s_{2}$ that is transformed into an appropriate range of $x_{1}$. It can be understood that the condition $s_{2}=0$ of subsystem $\mathrm{B}$ can be integrated into $s_{1}$ via $z$; therefore, the two subsystems can be controlled simultaneously by one control input.

The time derivation of (5) is

$$
\dot{s}_{1}=\lambda_{1} \gamma_{1}\left|x_{1}-z\right|^{\gamma_{1}-1}\left(\dot{x}_{1}-\dot{z}\right)+\dot{x}_{2},
$$

and the following equation can be obtained by setting (9) equal to zero

$$
\dot{x}_{2}+\lambda_{1} \gamma_{1}\left|x_{1}-z\right|^{\gamma_{1}-1} x_{2}=\lambda_{1} \gamma_{1}\left|x_{1}-z\right|^{\gamma_{1}-1} \dot{z} .
$$

The solution of (10) is as follows:

$$
x_{2}(t)=x_{2}(0) e^{-\lambda_{1} \gamma_{1} \int\left|x_{1}-z\right|^{\gamma_{1}-1} \mathrm{~d} t}+\lambda_{1} \gamma_{1} e^{-\lambda_{1} \gamma_{1} \int\left|x_{1}-z\right|^{\gamma_{1}-1} \mathrm{~d} t} \int e^{\lambda_{1} \gamma_{1} \int\left|x_{1}-z\right|^{\gamma_{1}-1} \mathrm{~d} t}\left|x_{1}-z\right|^{\gamma_{1}-1} \dot{z} \mathrm{~d} t^{\prime}
$$

where $x_{2}(0)$ is the initial value of $x_{2}(t) . x_{2}(t)$ converges to zero when $x_{1}=z$ and $\dot{z}=0 \dot{z}=0$; in other words, the control objective $\left[\begin{array}{ll}x_{10} & x_{20}\end{array}\right]^{\mathrm{T}}=\left[\begin{array}{ll}z & 0\end{array}\right]^{\mathrm{T}}$ can be achieved. Also, $z$ converges to zero which ensures that states $x_{1}$ and $x_{2}$ converge to the equilibrium points $\left[\begin{array}{ll}x_{10} & x_{20}\end{array}\right]^{\mathrm{T}}=\left[\begin{array}{ll}0 & 0\end{array}\right]^{\mathrm{T}^{2}}$. In the following section, the control method is proposed to guarantee that the sliding surfaces $s_{1}$ and $s_{2}$ converge to zero.

As we all know, the control input $u$ in (2) and (4) consists of the equivalent control law $u_{\mathrm{eq}}$ and the switching control law $u_{\text {sw }}$; in the next subsection, the extraction of $u_{\text {sw }}$ will be described. Here, according to the equivalent control method, when (9) is equal to zero, i.e., $\dot{s}_{1}=0$, substituting (2), $u_{\mathrm{eq}}$ can be calculated.

$$
u_{\mathrm{eq}}=\frac{-\lambda_{1} \gamma_{1}\left|x_{1}-z\right|^{\gamma_{1}-1}\left(\dot{x}_{1}-\dot{z}\right)-f_{1}(\mathbf{x})}{b_{1}(\mathbf{x})} .
$$

2.1.2. Piecewise Double Power Reaching Law. On the chattering caused by SMC, the DPRL can suppress the chattering, but the convergence speed is slow. Therefore, in order to improve the convergence speed of the system and reduce the chattering, a novel PDPRL with the characteristics of fixed-time convergence is designed, and different stages of the reaching law can be chosen separately without affecting each other.

The PDPRL is devised as

$\dot{s_{1}}=\left\{\begin{array}{l}-k_{1}\left|s_{1}\right|^{\alpha_{1}} \operatorname{sign}\left(s_{1}\right)-k_{2}\left|s_{1}\right|^{\alpha_{2}} \operatorname{sign}\left(s_{1}\right)-k s_{1} \quad\left|s_{1}\right| \geq 1 \\ -k_{3}\left|s_{1}\right|^{\beta_{1}} \operatorname{sign}\left(s_{1}\right)-k_{4}\left|s_{1}\right|^{\beta_{2}} \operatorname{sign}\left(s_{1}\right) \quad\left|s_{1}\right|<1\end{array}\right.$,

where $k, k_{1}, k_{2}, k_{3}$, and $k_{4}$ are positive constants, $k+k_{1}+k_{2}=k_{3}+k_{4}, \quad k_{3}=\left\{\begin{array}{ll}\varepsilon_{1} & 1 / e \leq\left|s_{1}\right|<1 \\ 0 & \left|s_{1}\right|<1 / e\end{array}\right.$, $k_{4}=\left\{\begin{array}{ll}\varepsilon_{2} & 1 / e \leq\left|s_{1}\right|<1 \\ \varepsilon_{1}+\varepsilon_{2} & \left|s_{1}\right|<1 / e\end{array}, \quad e \approx 2.71828, \quad \varepsilon_{1}>0, \quad \varepsilon_{2}>0\right.$, $0<\alpha_{1}<1, \alpha_{2}>1, \beta_{1}>1$, and $0<\beta_{2}<1$.

The main idea of the PDPRL is as follows: taking 1 as the demarcation point, the reaching process is divided into two stages, far from the sliding mode surface, i.e., $\left|s_{1}\right| \geq 1$, and near the sliding mode surface, i.e., $\left|s_{1}\right|<1$. When the system is far from the sliding mode surface, the reaching law is dominated by the first term, and when it is near the sliding mode surface, the reaching law is dominated by the second term. The property of the power function is used to make the 
system have a large approaching rate at both stages, and the reaching law is equal to zero at the equilibrium point $\left(\left|s_{1}\right|=0\right)$. The condition $k+k_{1}+k_{2}=k_{3}+k_{4}$ must be satisfied to ensure continuity of the reaching law. In addition, the reaching law that dominates $\left|s_{1}\right| \geq 1$ and $\left|s_{1}\right|<1$ can be designed separately, and the two stages will not affect each other.

(1) Fixed-Time Convergence. For the reaching law (13) proposed above, assuming that $s_{10}$ is the initial value of $s_{1}$, if the condition $\alpha_{1}+\alpha_{2}=\beta_{1}+\beta_{2}=2$ is satisfied, in a fixed time, $s_{1}$ can converge to zero. The maximum convergence time $T$ exists and is not concerned with the initial value $s_{10}$. The convergence time of each stage is rough as follows.

Suppose $s_{1}>0$; if $s_{10} \geq 1$, the approaching process is separated into two stages: $s_{10} \longrightarrow 1$ and $1 \longrightarrow 0$. When $1 \leq s_{1}<s_{10}$, (13) can be written as $\dot{s_{1}}=-k_{1}\left|s_{1}\right|^{\alpha_{1}}-k_{2}\left|s_{1}\right|^{\alpha_{2}}-\mathrm{ks}_{1}$. It is difficult to solve the equation; then, divide it into two equations, $\dot{s}_{1}+k_{2}\left|s_{1}\right|^{\alpha_{2}}+$ $\mathrm{ks}_{1}=0$ and $\dot{s}_{1}+k_{1}\left|s_{1}\right|^{\alpha_{1}}+\mathrm{ks}_{1}=0$, and solve them separately. Since the solutions of the two equations represent the reaching time required under the influence of $-k_{2}\left|s_{1}\right|^{\alpha_{2}} \operatorname{sign}\left(s_{1}\right)$ and $-k_{1}\left|s_{1}\right|^{\alpha_{1}} \operatorname{sign}\left(s_{1}\right)$, the approaching time must be less than either of the two solutions. Here, the convergence time required under the influence of $-k_{2}\left|s_{1}\right|^{\alpha_{2}} \operatorname{sign}\left(s_{1}\right)$ can be calculated.

$$
\dot{s_{1}}+k_{2} s_{1}^{\alpha_{2}}+\mathrm{ks}_{1}=0
$$

with $s_{1}=1$; the convergence time $T_{1}$ of (14) can be obtained as

$$
T_{1}=\frac{1}{k\left(\alpha_{2}-1\right)}\left[\ln \left(k+k_{2}\right)-\ln \left(\mathrm{ks}_{10}^{1-\alpha_{2}}+k_{2}\right)\right] .
$$

When $1 / e<s_{1}<1$, (13) can be written as

$$
\dot{s}_{1}+k_{3} s_{1}^{2-\beta_{2}}+k_{4} s_{1}^{\beta_{2}}=0
$$

with $s_{1}=1 / e$; the convergence time $T_{2}$ of (16) can be calculated as

$$
\begin{aligned}
& T_{2}=\frac{\arctan \left[\sqrt{k_{3} / k_{4}}\left|s_{10}\right|^{1-\beta_{2}}\left(1-e^{\beta_{2}-1}\right) /\left(1+k_{3} / k_{4} e^{\beta_{2}-1}\left|s_{10}\right|^{1-\beta_{2}}\right)\right]}{\sqrt{k_{3} k_{4}}\left(1-\beta_{2}\right)} . \\
& \qquad T_{3}=\frac{1}{k_{4}}\left(1-\beta_{2}\right) .
\end{aligned}
$$

When $0<s_{1}<1 / e$, (13) can be written as

$$
\dot{s}_{1}+k_{4} s_{1}^{\beta_{2}}=0,
$$

with $s_{1}=0$, the convergence time $T_{3}$ is
When $0<s_{10}<1$, similar to the process $1 / e<s_{1}<1$ and $0<s_{1}<1 / e$ described above, the convergence time $T_{4}$ is as follows

$$
T_{4}=T_{2}+T_{3}=\frac{\arctan \left[\sqrt{k_{3} / k_{4}}\left|s_{10}\right|^{1-\beta_{2}}\left(1-e^{\beta_{2}-1}\right) /\left(1+k_{3} / k_{4} e^{\beta_{2}-1}\left|s_{10}\right|^{1-\beta_{2}}\right)\right]}{\sqrt{k_{3} k_{4}}\left(1-\beta_{2}\right)+1 / k_{4}\left(1-\beta_{2}\right)} .
$$

Because $T_{1}$ in (15) has a maximum value as follows:

$$
T_{1}=\frac{1}{k\left(\alpha_{2}-1\right)}\left[\ln \left(k+k_{2}\right)-\ln \left(\mathrm{ks}_{10}^{1-\alpha_{2}}+k_{2}\right)\right] \leq \frac{1}{k\left(\alpha_{2}-1\right)}\left(\frac{k+k_{2}}{\mathrm{ks}_{10}^{1-\alpha_{2}}+k_{2}}-1\right)=\frac{1-s_{10}^{1-\alpha_{2}}}{\left(\alpha_{2}-1\right)\left(\mathrm{ks}_{10}^{1-\alpha_{2}}+k_{2}\right)}<\frac{1}{k_{2}\left(\alpha_{2}-1\right)}
$$

$\arctan (\cdot)<\pi / 2$. Thus, the convergence time is

$$
T= \begin{cases}T_{1}+T_{2}+T_{3}<\frac{1}{k_{4}}\left(1-\beta_{2}\right)+\frac{1}{k_{2}}\left(\alpha_{2}-1\right)+\frac{\pi}{2} \sqrt{k_{3} k_{4}}\left(1-\beta_{2}\right), & \left|s_{10}\right| \geq 1, \\ T_{3}+T_{4}<\frac{2}{k_{4}}\left(1-\beta_{2}\right)+\frac{\pi}{2} \sqrt{k_{3} k_{4}}\left(1-\beta_{2}\right), & \left|s_{10}\right|<1 .\end{cases}
$$


The result above is obtained in the case of $s_{1}>0$, and the result of $s_{1}<0$ is similar to the above, which is not repeated here.
Then, the switching control law $u_{s w}$ is

$$
u_{\mathrm{sW}}= \begin{cases}-\frac{1}{b_{1}(x)}\left(k_{1}\left|s_{1}\right|^{\alpha_{1}} \operatorname{sign}\left(s_{1}\right)+k_{2}\left|s_{1}\right|^{\alpha_{2}} \operatorname{sign}\left(s_{1}\right)+\mathrm{ks}_{1}\right), & \left|s_{1}\right| \geq 1, \\ -\frac{1}{b_{1}(x)}\left(k_{3}\left|s_{1}\right|^{\beta_{1}} \operatorname{sign}\left(s_{1}\right)+k_{4}\left|s_{1}\right|^{\beta_{2}} \operatorname{sign}\left(s_{1}\right)\right), & \left|s_{1}\right|<1,\end{cases}
$$

and the control strategy $u$ of the whole system without disturbance can be obtained as

$$
u=u_{\mathrm{eq}}+u_{\mathrm{sw}}
$$

2.2. PDPRL-DTSMC with Disturbance. Most control methods are based on the accurate system model, but it is difficult to achieve in practical industrial applications, and the actual situation may be more complex [38]; there are many unknown external disturbances in the system, and the parameters of the system may change with the change of time and environment, which requires the robustness of the control method. Therefore, the ubiquitous external disturbances and model parameter uncertainties of the system are considered as lumped disturbances, and the disturbance observer is designed.

Here, only the lump disturbance in subsystem A is considered, and the nonlinear underactuated system (1)-(4) can be reformulated as

$$
\left\{\begin{array}{l}
\dot{x}_{1}=x_{2} \\
\dot{x}_{2}=f_{1}(\mathbf{x})+b_{1}(\mathbf{x}) u+d \\
\dot{x}_{3}=x_{4} \\
\dot{x}_{4}=f_{2}(\mathbf{x})+b_{2}(\mathbf{x}) u
\end{array}\right.
$$

where $d$ is the lump disturbance and the time derivative of the lump disturbance is supposed to be bounded, and $|\dot{d}| \leq D, D$ is a positive constant.
Considering the lack of the ability of DTSMC to suppress the disturbance, an STDO is adopted to estimate the lump disturbance, also an estimated value is compensated to the controller to weaken the influence of disturbance on the system. Figure 1 presents the control method diagram.

The STDO is constructed for subsystem A as follows [17]:

$$
\begin{aligned}
\left\{\dot{\hat{x}}_{2}\right. & =f_{1}(\mathbf{x})+b_{1}(\mathbf{x}) u+\widehat{d}, \\
\widehat{d} & =a\left|\widetilde{x}_{2}\right|^{1 / 2} \operatorname{sign}\left(\widetilde{x}_{2}\right)-\varepsilon, \\
\dot{\varepsilon} & =-b \operatorname{sign}\left(\widetilde{x}_{2}\right),
\end{aligned}
$$

where $\dot{\hat{x}}_{2}$ is the time derivative of $\widehat{x}_{2}$ which is the estimate of state $x_{2} \cdot \hat{d}$ is the estimate of disturbance $d . a$ and $b$ are positive numbers that need to be designed. $\tilde{x}_{2}$ is the error, which is defined as $\widetilde{x}_{2}=x_{2}-\widehat{x}_{2}$. $\dot{\varepsilon}$ is the time derivative of internal variable $\varepsilon$.

Substitute $\dot{\hat{x}}_{2}$ into (9):

$$
\dot{s}_{1}=\lambda_{1} \gamma_{1}\left|x_{1}-z\right|^{\gamma_{1}-1}\left(\dot{x}_{1}-\dot{z}\right)+\dot{\hat{x}}_{2} \text {. }
$$

Let the above equation be equal to zero, and using (26), $u_{\text {eq }}$ can be recalculated as

$$
u_{\text {eq }}=\frac{-\lambda_{1} \gamma_{1}\left|x_{1}-z\right|^{\gamma_{1}-1}\left(x_{2}-\dot{z}\right)-f_{1}(\mathbf{x})-\hat{d}}{b_{1}(\mathbf{x})} .
$$

The control input $u$ of the system is

$$
u= \begin{cases}-\frac{1}{b_{1}(\mathbf{x})}\left(\lambda_{1} \gamma_{1}\left|x_{1}-z\right|^{\gamma_{1}-1}\left(x_{2}-\dot{z}\right)+f_{1}(\mathbf{x})+\widehat{d}+k_{1}\left|s_{1}\right|^{\alpha_{1}} \operatorname{sign}\left(s_{1}\right)+k_{2}\left|s_{1}\right|^{\alpha_{2}} \operatorname{sign}\left(s_{1}\right)+\mathrm{ks}_{1}\right), & \left|s_{1}\right| \geq 1, \\ -\frac{1}{b_{1}(\mathbf{x})}\left(\lambda_{1} \gamma_{1}\left|x_{1}-z\right|^{\gamma_{1}-1}\left(x_{2}-\dot{z}\right)+f_{1}(\mathbf{x})+\widehat{d}+k_{3}\left|s_{1}\right|^{\beta_{1}} \operatorname{sign}\left(s_{1}\right)+k_{4}\left|s_{1}\right|^{\beta_{2}} \operatorname{sign}\left(s_{1}\right)\right), & \left|s_{1}\right|<1 .\end{cases}
$$

2.3. Stability Analysis. Through the analysis of the fixed-time convergence characteristics of the PDPRL in Section 2.1.2, it can be seen that the reaching law can slide to the sliding mode surfaces in fixed time and the convergence time is not concerned with the initial value $s_{10}$. Therefore, it ensures that the system using PDPRL can slide to the surfaces in fixed time no matter how far away it is from the surfaces.

Proposition 1. For the nonlinear underactuated system defined in equation (25) with $s_{1}$ and $s_{2}$ in equations (5) and 


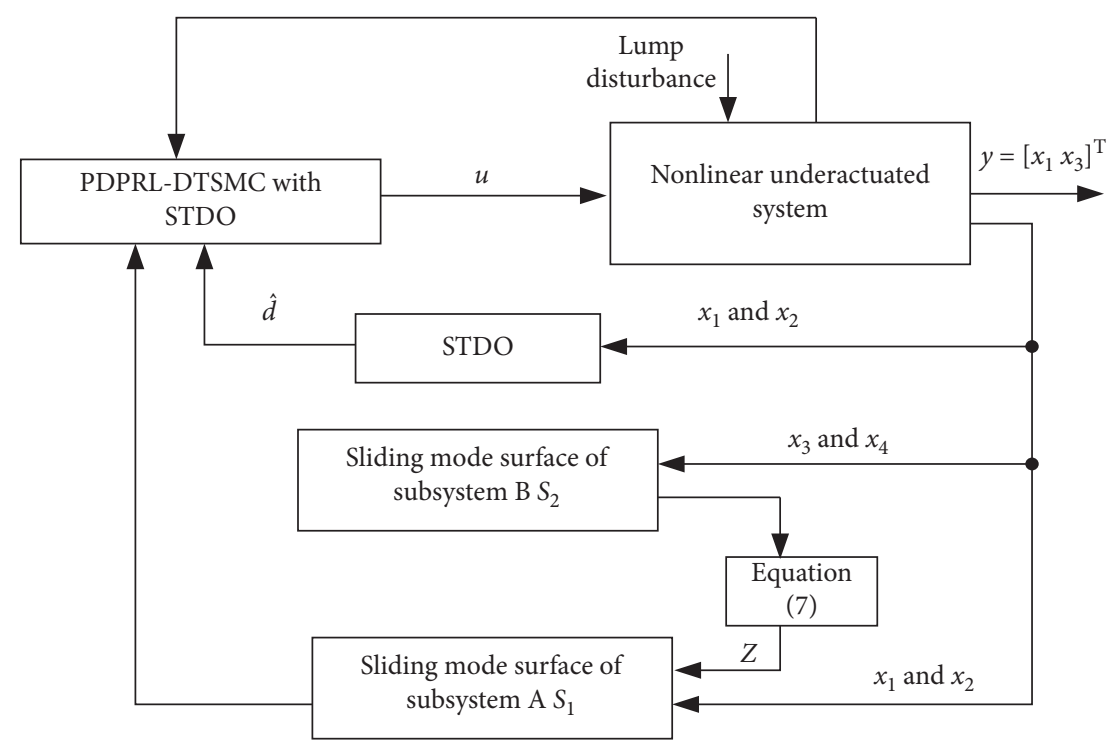

FIGURE 1: System control structure diagram.

(6), if the control input $u$ is devised as equation (29), then the system is ensured to be asymptotically stable.

Proof. The Lyapunov function is chosen as

$$
V(t)=\frac{1}{2} s_{1}^{2}
$$

Taking the time derivative of $V(t)$ and using (25) and (27), the following equation can be obtained:

$$
\begin{aligned}
\dot{V}(t)= & s_{1} \dot{s}_{1}=s_{1}\left(\lambda_{1} \gamma_{1}\left|x_{1}-z\right|^{\gamma_{1}-1}\left(x_{2}-\dot{z}\right)\right. \\
& \left.+f_{1}(\mathbf{x})+b_{1}(\mathbf{x}) u+\widehat{d}\right) .
\end{aligned}
$$

For the sake of proving conveniently, the control law of $\left|s_{1}\right| \geq 1$ is adopted, and substituting it into (31), then (31) can be written as follows:

$$
\dot{V}(t)=-\left(k_{1} s_{1}\left|s_{1}\right|^{\alpha_{1}} \operatorname{sign}\left(s_{1}\right)+k_{2} s_{1}\left|s_{1}\right|^{\alpha_{2}} \operatorname{sign}\left(s_{1}\right)+\mathrm{ks}_{1}^{2}\right)<0 .
$$

Since $k>0, k_{1}>0, k_{2}>0,0<\alpha_{1}<1$, and $\alpha_{2}>1$, whether the sign of $s_{1}$ is positive or negative, $\dot{V}(t)$ is always negative. In other words, system (25) is ensured to be asymptotically stable, and $s_{1}$ and $s_{2}$ can be reached. When $s_{1}=0$, i.e., subsystem $\mathrm{A}$ is on $s_{1}, x_{1}=z$ and $x_{2}=0$ can be obtained. According to equation (11), $x_{2} \longrightarrow 0$ if and only if $z \longrightarrow 0$; from equation (7), $s_{2} \longrightarrow 0$ is ensured. Therefore, the two subsystems can be stabilized with one control input.

\section{Example and Result Analysis}

The proposed control strategy, i.e., DTSMC strategy based on novel reaching law and disturbance observer, is simulated to verify the effectiveness in this section. The simulation verification and comparison are implemented on Matlab/ Simulink platform.

The inverted pendulum system is a typical underactuated system with strong nonlinearity, strong coupling, and natural instability, which is widely used in the fields of a rocket launch, satellite attitude control, and biped robots. Many typical problems in control theory, such as tracking control problems, robust control problems, and nonlinear control problems, are included in the inverted pendulum system. Therefore, the inverted pendulum system is taken as an example to illustrate that the control strategy is correct.

The physical model of the inverted pendulum system is exhibited in Figure 2, its dynamic equation can be expressed by (25), and $f_{1}(\mathbf{x}(t)), f_{2}(\mathbf{x}(t)), b_{1}(\mathbf{x}(t))$, and $b_{2}(\mathbf{x}(t))$ are as follows.

$f_{1}(\mathbf{x}(t))=\frac{\left(m_{1}+m_{2}\right) g \sin \left(x_{1}\right)-m_{1} L \sin \left(x_{1}\right) \cos \left(x_{1}\right) x_{2}^{2}}{L\left((4 / 3)\left(m_{1}+m_{2}\right)-m_{1} \cos ^{2}\left(x_{1}\right)\right)}$,

$b_{1}(\mathbf{x}(t))=\frac{\cos \left(x_{1}\right)}{L\left((4 / 3)\left(m_{1}+m_{2}\right)-m_{1} \cos ^{2}\left(x_{1}\right)\right)}$,

$f_{2}(\mathbf{x}(t))=\frac{-(4 / 3) m_{1} \mathrm{Lx}_{2}^{2} \sin \left(x_{1}\right)+m_{1} g \sin \left(x_{1}\right) \cos \left(x_{1}\right)}{(4 / 3)\left(m_{1}+m_{2}\right)-m_{1} \cos ^{2}\left(x_{1}\right)}$,

$b_{2}(\mathbf{x}(t))=\frac{4}{3\left((4 / 3)\left(m_{1}+m_{2}\right)-m_{1} \cos ^{2}\left(x_{1}\right)\right)}$,

where $x_{1}$ is the angle between the pendulum and upright direction; $x_{2}$ and $x_{4}$ are the angular velocity of the pendulum and velocity of the cart, respectively. $x_{3}$ is the distance from the cart to the origin. $L$ is the length from the center point of the pendulum to the cart. $m_{1}$ and $m_{2}$ are the mass of the pendulum and the cart, respectively. $u$ is the force to the cart. $x_{1}$ and $x_{2}$ are selected as the primary target, subsystem $\mathrm{A}$, and $x_{3}$ and $x_{4}$ as subsystem $\mathrm{B}$, the secondary target. $x(0)=$ $\left[\begin{array}{cccc}-60^{\circ} & 0 & 0 & 0\end{array}\right]^{T}$ is selected as the initial state, and the parameters adopted here are the same as those in [20], $\mathrm{g}=9.8 \mathrm{~ms}^{2}, L=0.5 \mathrm{~m}, m_{1}=0.05 \mathrm{~kg}$, and $m_{2}=1 \mathrm{~kg}$. 


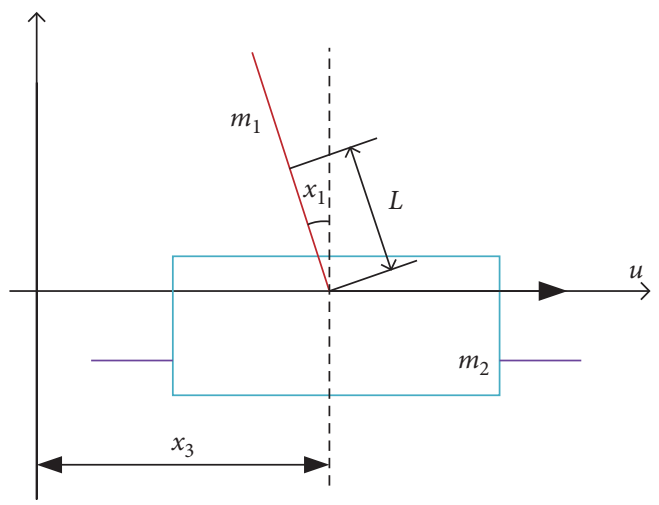

Figure 2: Inverted pendulum system.

When the system states are far from the sliding mode surface $\left|s_{1}\right| \geq 1$, increasing $k, k_{1}, k_{2}, \alpha_{1}$, and $\alpha_{2}$ can increase the reaching speed and shorten the time to reach the desired position. When the system states are close to the sliding mode surface $\left|s_{1}\right|<1$, increasing $k_{3}, k_{4}, \beta_{1}$, and $\beta_{2}$ can also increase the reaching speed, but the too large parameter values will increase the chattering and steady-state error of the system. Through the empirical trial and error method and a large number of simulations, the specific parameters are determined. As shown in Figure 3, with the decrease of $\lambda_{1}$, the reaching speed of the system will be accelerated, the time to reach the sliding mode surface will be shortened, but the chattering of the system will be increased; with the increase of $\lambda_{2}$, the reaching speed of the system will not change significantly, but it will increase the overshoot of the system. The parameters are chosen as follows: $k=2, k_{1}=2$, $k_{2}=6, \varepsilon_{1}=\varepsilon_{2}=5, r=2, r_{1}=\beta_{2}=0.4, \alpha_{1}=0.3, \alpha_{2}=1.7$, $\beta_{1}=1.6, \lambda_{1}=5, \lambda_{2}=0.8, a=0.6$, and $b=1$.

3.1. Characteristic Simulation of PDPRL. The characteristic of PDPRL is simulated in a system with single input single output.

$$
\dot{s}=u,
$$

where $s$ and $u$ are the output and control input, respectively. $\dot{s}$ is the time derivative of the sliding mode variable $s . s_{0}=50$, $s_{0}=5$, and $s_{0}=1$ are defined as the three initial values of the sliding mode variable. Compared with FPRL and DPRL, the proposed PDPRL is simulated to test the fixed-time convergence characteristic. The expression for the reaching law used above is as follows.

(1) FPRL

$$
u_{1}=-k s-k_{2}|s|^{r_{1}} \operatorname{sign}(s) .
$$

(2) DPRL

$$
u_{2}=-k_{1}|s|^{r} \operatorname{sign}(s)-k_{2}|s|^{r_{1}} \operatorname{sign}(s) .
$$

(3) PDPRL

$u_{3}=\left\{\begin{array}{l}-k_{1}|s|^{\alpha_{1}} \operatorname{sign}(s)-k_{2}|s|^{\alpha_{2}} \operatorname{sign}(s)-\mathrm{ks}, \quad|s| \geq 1, \\ -k_{3}|s|^{\beta_{1}} \operatorname{sign}(s)-k_{4}|s|^{\beta_{2}} \operatorname{sign}(s), \quad|s|<1 .\end{array}\right.$

Under the condition of $s_{0}=50, s_{0}=5$, and $s_{0}=1$, Figure 4 presents the simulation results of the three reaching laws.

As shown in Figure 4, when $s_{0}=1$, the speed for the proposed reaching law to slide to the sliding surface is relatively slow, but still fast. When $s_{0}=5$ and $s_{0}=50$, its approaching speed is the fastest and the time to the equilibrium point is the shortest. It is also obvious that with the increase of $s_{0}$, the convergence time of FPRL and DPRL is prolonged; when $s_{0}=1, s_{0}=5$, and $s_{0}=50$, the convergence time of FPRL and DPRL is $0.18 \mathrm{~s}, 0.41 \mathrm{~s}$, and $1.3 \mathrm{~s}$ and $0.18 \mathrm{~s}$, $0.37 \mathrm{~s}$, and $0.51 \mathrm{~s}$, respectively, while the convergence time of PDPRL is $0.24 \mathrm{~s}, 0.32 \mathrm{~s}$, and $0.5 \mathrm{~s}$. Therefore, the three reaching laws achieve the convergence of the sliding mode variable to the equilibrium point under different initial conditions. But the rate of convergence is different, clearly, the rate of convergence for the devised PDPRL is the fastest, and the maximum convergence time is not affected by $s_{0}$, which corresponds to the fixed-time convergence in Section 2.1.2.

3.2. Simulation Results and Analysis of the Inverted Pendulum System. In this section, the proposed control strategy is applied to the inverted pendulum system with or without the lump disturbance, and the simulation and analysis are carried out, respectively.

3.2.1. System without Disturbance. When the disturbance of the system is not considered, i.e., $d=0$, the simulation results are demonstrated in Figures 5-7.

By comparing FPRL, DPRL, and the proposed PDPRL with DTSMC, the angular position response curves of the 


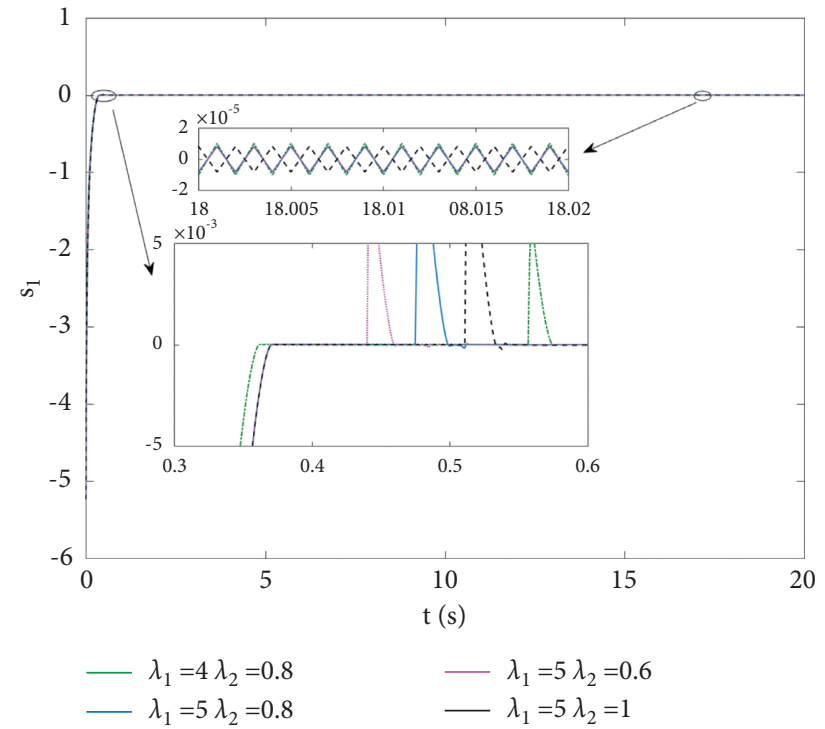

Figure 3: Sliding mode surface for subsystem A.

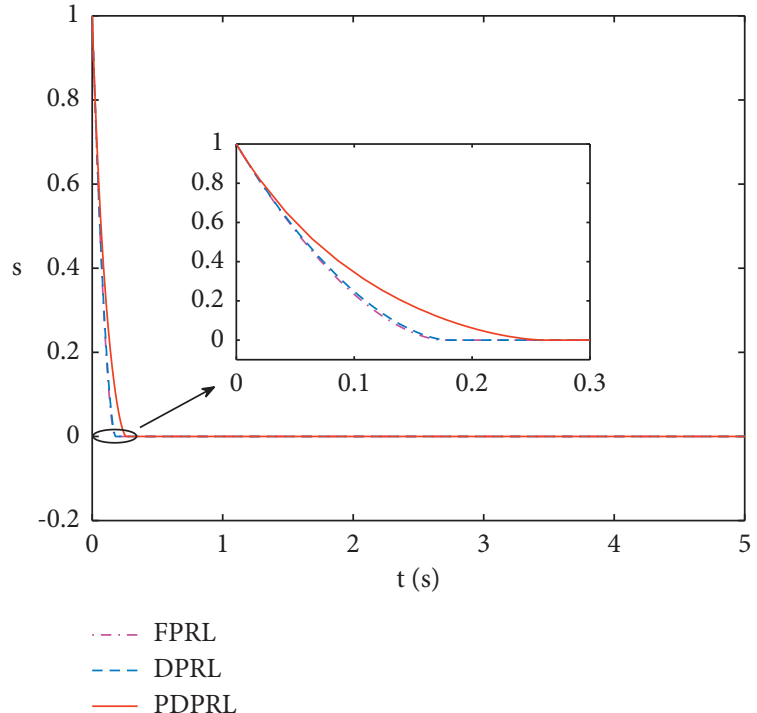

(a)

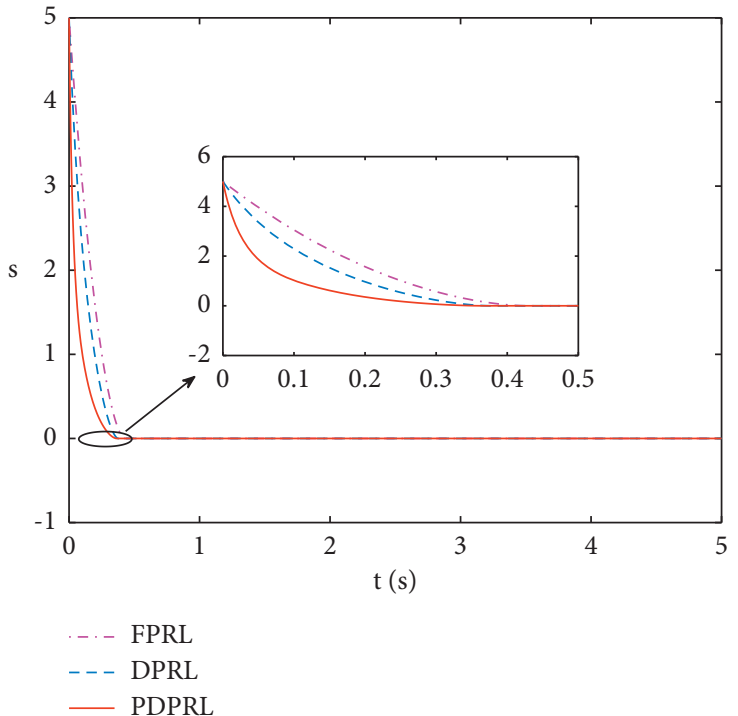

(b)

Figure 4: Continued. 


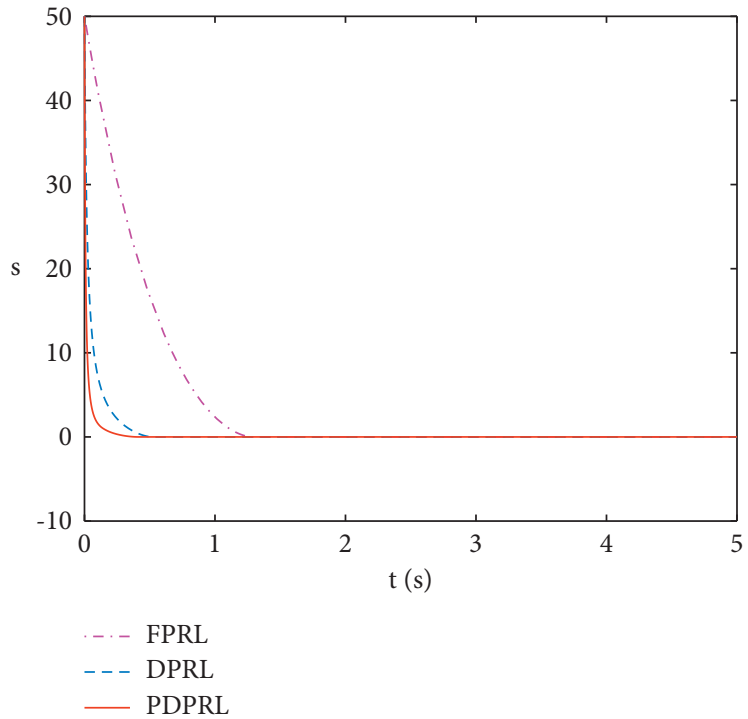

(c)

FIgURE 4: $s$ convergence curves with different $s_{0}$. (a) $s_{0}=1$. (b) $s_{0}=5$. (c) $s_{0}=50$.
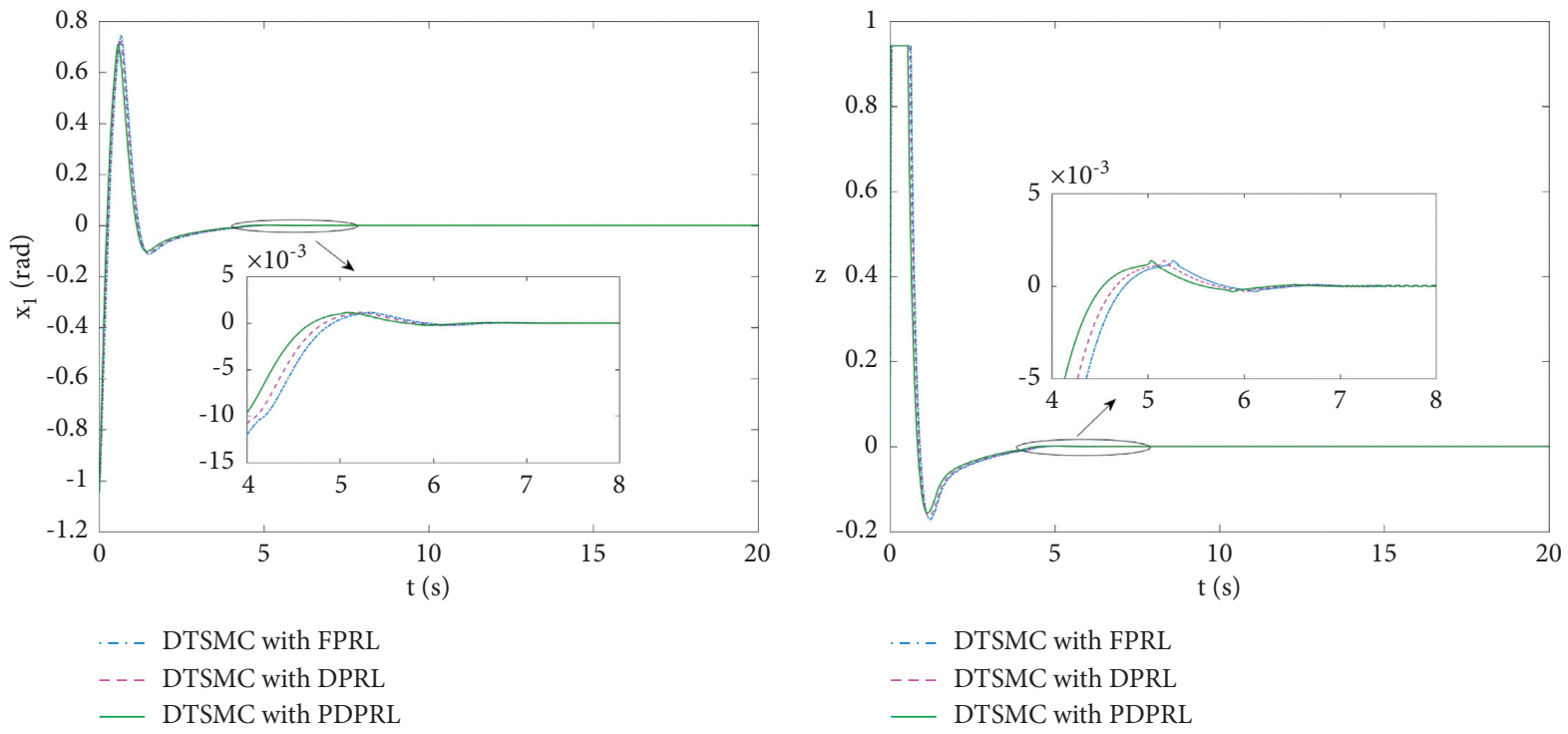

(a)

(b)

Figure 5: Continued. 


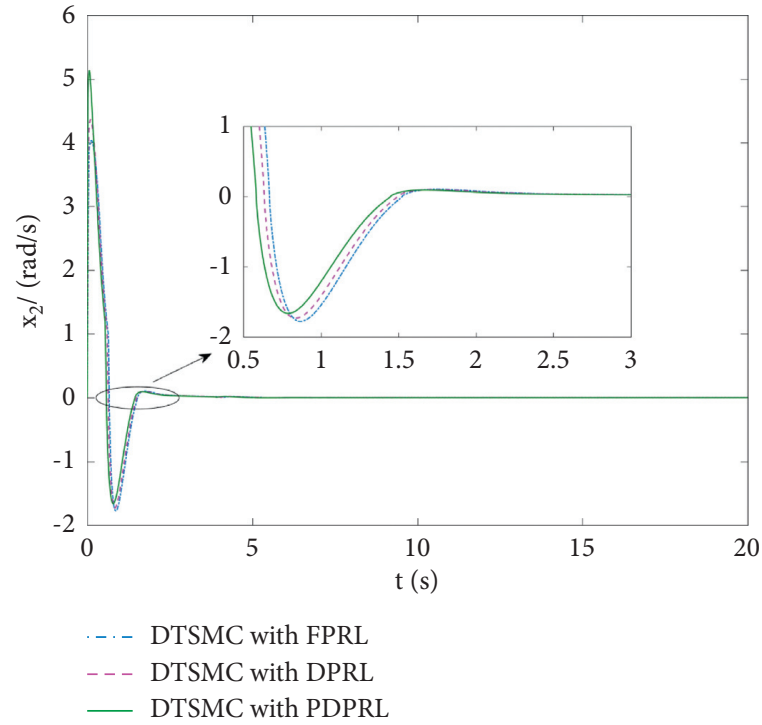

(c)

Figure 5: Simulation of DTSMC based on FPRL, DPRL, and PDPRL. (a) Angular position of the pendulum. (b) Intermediate signal $z$. (c) Angular velocity of the pendulum. (d) Position of the cart.

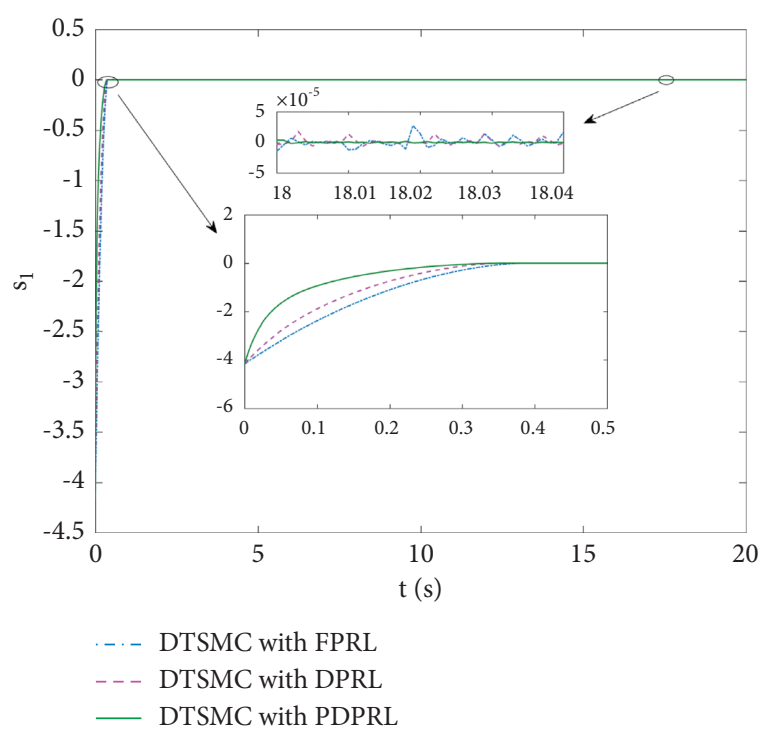

(a)

Figure 6: Simulation of DTSMC based on FPRL, DPRL, and PDPRL. (a) Sliding mode surface for subsystem A. (b) Sliding mode surface for subsystem $B$.

pendulum are exhibited in Figure 5(a). Obviously, the proposed strategy shows a shorter convergence time to the equilibrium point than DTSMC with FPRL and DPRL, the time is $6.2 \mathrm{~s}, 6.5 \mathrm{~s}$, and $6.4 \mathrm{~s}$, respectively, and it has a minor overshoot. Similarly, this feature can be seen from the response curves of $z$ shown in Figure 5(b). The angular velocity response curves of the pendulum and the position response curves of the cart are shown in Figures 5(c) and 5(d), respectively. Clearly, the proposed control method can quickly stabilize the cart in the equilibrium position of about $6.5 \mathrm{~s}$

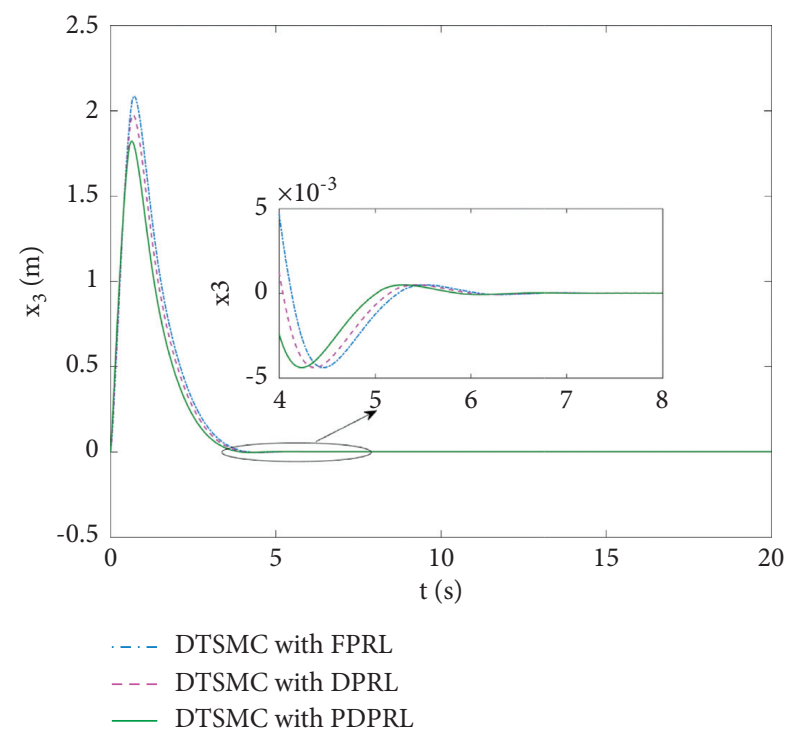

(d)

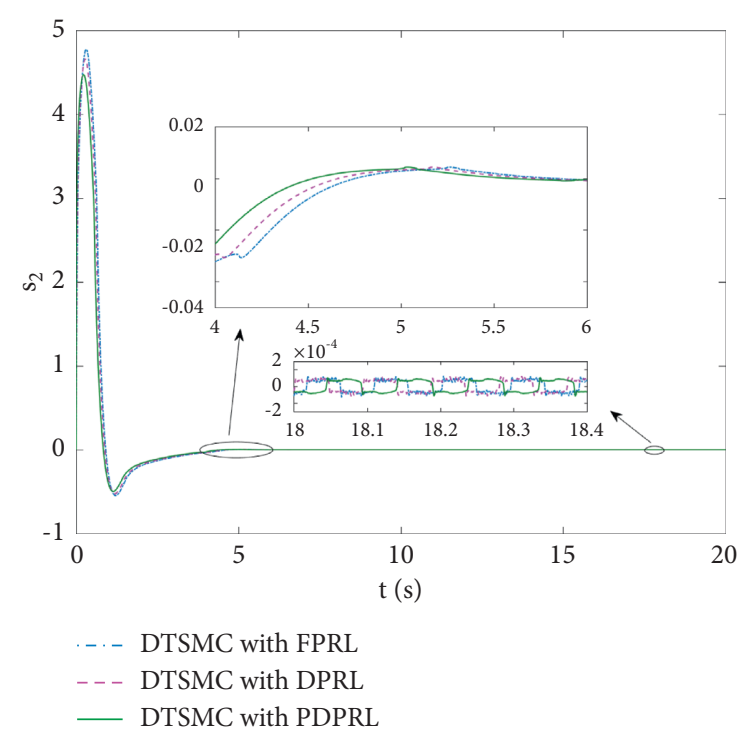

(b) and the control method using FPRL and DPRL takes $6.7 \mathrm{~s}$ and $6.9 \mathrm{~s}$, respectively. The sliding mode surface $s_{1}$ of subsystem A is shown in Figure 6(a); the response speed of the system with the proposed control strategy reach sliding mode surface is fast; as can be seen in the sliding mode surface of subsystem B shown in Figure 6(b), about $0.34 \mathrm{~s}$ and the chattering that occurs in the system can be effectively reduced, which proves that the devised strategy can reduce chattering. Although the response speed of the control method with FPRL and DPRL is also fast, about $0.34 \mathrm{~s}$ and 


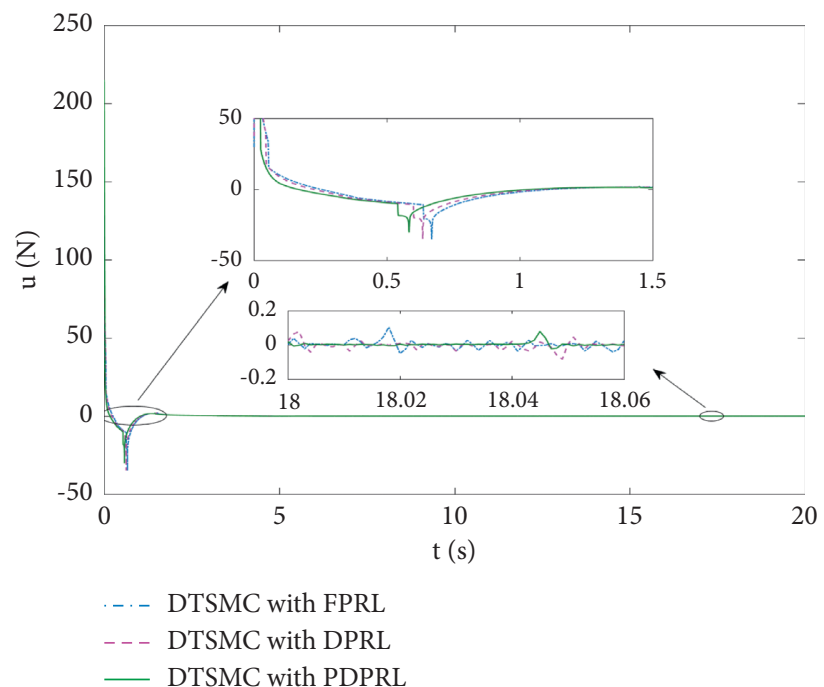

FIgURe 7: Control input with DTSMC based on FPRL, DPRL, and PDPRL.
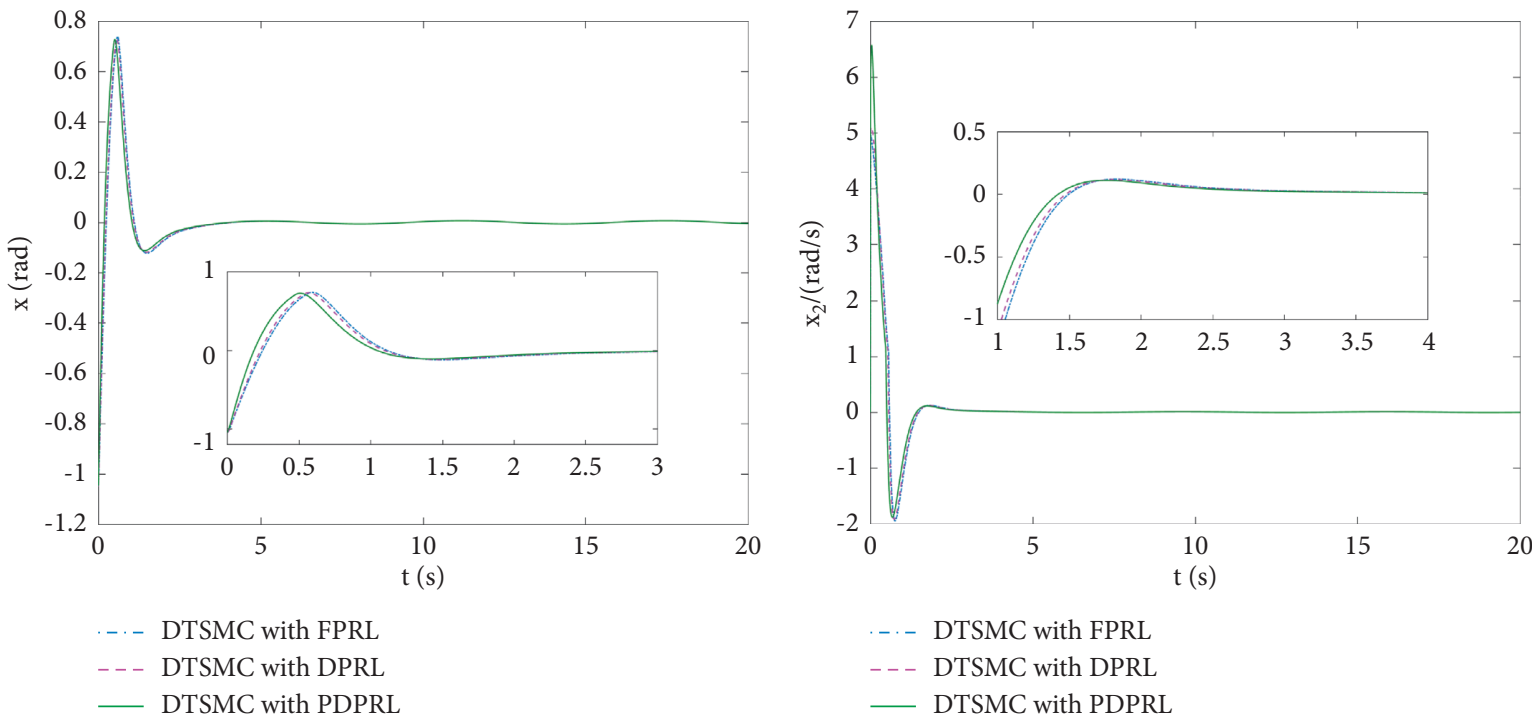

(a)

(b)

Figure 8: Continued. 


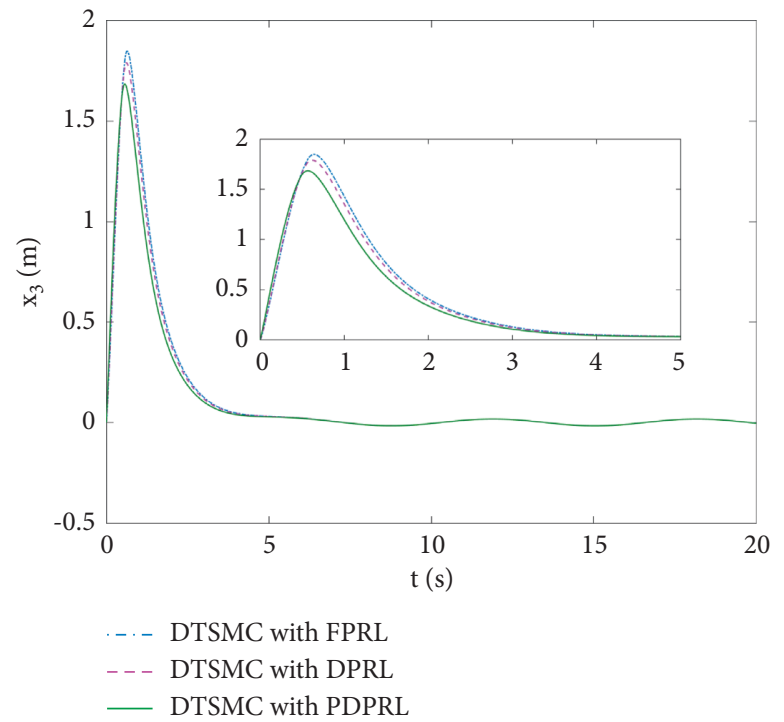

(c)

Figure 8: Simulation of DTSMC based on FPRL, DPRL, and PDPRL with STDO. (a) Angular position of the pendulum. (b) Angular velocity of the pendulum. (c) Position of the cart.

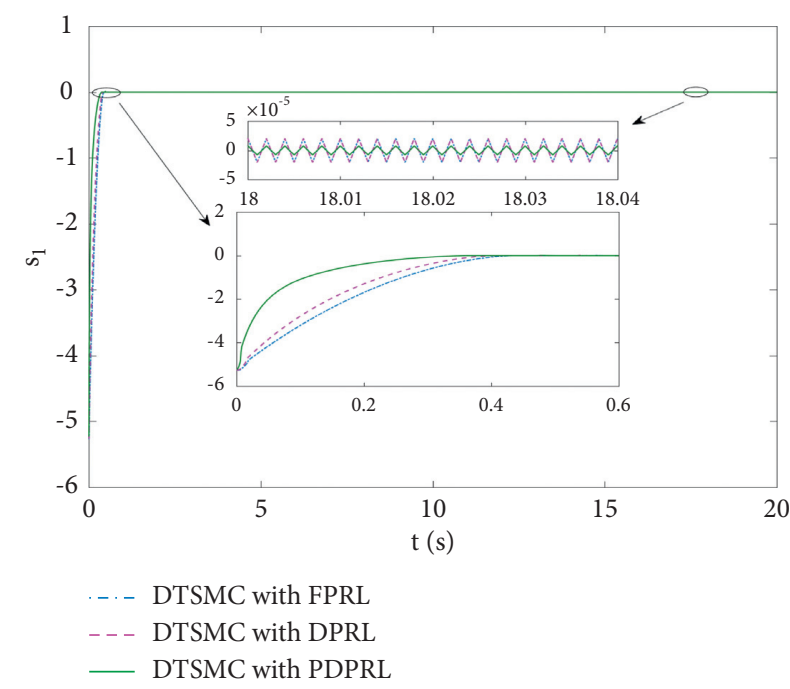

FIGURE 9: Sliding mode surface for subsystem A based on DTSMC with STDO.

$0.37 \mathrm{~s}$, respectively; the chattering is large. The control input is presented in Figure 7; clearly, the proposed strategy produces a faster input and smaller chattering.

3.2.2. System with Disturbance. Considering a lump disturbance in the subsystem A of system (25) and the disturbance is set to $d=0.2 \sin (t)$, Figures $8-10$ exhibit the simulation results.

The angular position and angular velocity response curves of the pendulum are demonstrated in Figures 8(a) and 8 (b), respectively. Figure 8(c) reveals the position curves of the cart. The response curves of $s_{1}$ are shown in Figure 9, the sliding mode surface has an overshoot, but the chattering

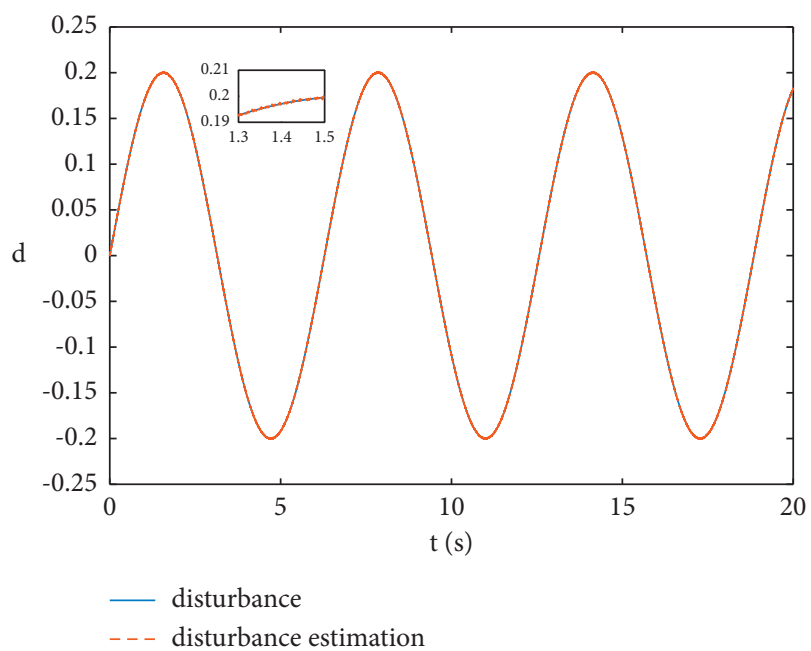

Figure 10: Disturbance estimation.

is small. As can be seen from Figure 10, the STDO can accurately estimate the disturbance. Those show that the estimated value can be compensated to the controller; the proposed method with STDO can reduce the disturbance effectively and have a shorter settling time.

Through the simulation and comparative analysis above, it can be seen that the proposed control strategy can reduce system chattering, increase the reaching speed of the system, shorten the time for the system to reach the desired position, and enhance the antidisturbance ability of the system.

\section{Conclusions}

A composite SMC strategy combining novel PDPRL and disturbance observer for a class of fourth-order nonlinear underactuated systems is proposed. A novel PDPRL based 
on piecewise function is devised to suppress the chattering of the system caused by SMC, and the fixed-time convergence characteristic and the maximum convergence time independent of initial value are analyzed. In addition, an STDO, which can accurately estimate the disturbance, is adopted to weaken the effect of the lump disturbance on the system. The proposed strategy is validated and easy to implement in the inverted pendulum system, and the controller parameters have certain regularity and are easy to adjust. By comparing with the control method using FPRL and DPRL, the simulation results show that the designed control strategy can improve the response speed, effectively reduce chattering, and enhance the robustness of the system.

4.1. Future Research Work. In this paper, the lump disturbance of subsystem A of the fourth-order nonlinear underactuated system is considered. On this basis, the lump disturbances of the whole nonlinear underactuated system will be considered and a more general disturbance suppression control method will be devised in the future. At the same time, the underactuated system with higher order will be considered, and a universal control strategy will be designed.

\section{Data Availability}

The data used to support the findings of this study are included within the article.

\section{Conflicts of Interest}

The authors declare that there are no conflicts of interest.

\section{Acknowledgments}

This research was funded by National Natural Science Foundation of China, Grant 61573203, and Natural Science Foundation of Shandong Province, Grant ZR2021MF005.

\section{References}

[1] M. Reyhanoglu, A. van der Schaft, N. H. Mcclamroch, and I. Kolmanovsky, "Dynamics and control of a class of underactuated mechanical systems," IEEE Transactions on Automatic Control, vol. 44, no. 9, pp. 1663-1671, 1999.

[2] S. Roy and S. Baldi, "Towards structure-independent stabilization for uncertain underactuated euler-Lagrange systems," Automatica, vol. 113, Article ID 108775, 2020.

[3] L. Chen and M. Van, "Sliding mode control of a class of underactuated system with non-integrable momentum," Journal of the Franklin Institute, vol. 357, no. 14, pp. 9484$9504,2020$.

[4] X. Chen, H. Zhao, H. Sun, S. Zhen, and K. Huang, "A novel adaptive robust control approach for underactuated mobile robot," Journal of the Franklin Institute, vol. 356, no. 5, pp. 2474-2490, 2019.

[5] T. Chen and B. Goodwine, "Controllability and accessibility results for n-link horizontal planar manipulators with one unactuated joint," Automatica, vol. 125, no. 5, pp. 1-15, 2021.

[6] S. Gong, A. Zhang, Z. Liu, Z. Li, C. Yang, and X. Zhang, "Nonlinear global stabilization control for the underactuated
WAcrobot system," Mathematical Problems in Engineering, vol. 2020, Article ID 3920535, 8 pages, 2020.

[7] B. K. Sahu, B. Subudhi, and M. M. Gupta, "Stability analysis of an underactuated autonomous underwater vehicle using extended-Routh's stability method," International Journal of Automation and Computing, vol. 15, no. 3, pp. 299-309, 2018.

[8] Z. Huang, X. Lai, P. Zhang, Q. Meng, and M. Wu, "A general control strategy for planar 3-dof underactuated manipulators with one passive joint," Information Sciences, vol. 534, pp. 139-153, 2020.

[9] M. Ryalat and D. S. Laila, "A robust IDA-PBC approach for handling uncertainties in underactuated mechanical systems," IEEE Transactions on Automatic Control, vol. 63, no. 10, pp. 3495-3502, 2018.

[10] R. Ortega, M. W. Spong, F. Gomez-Estern, and G. Blankenstein, "Stabilization of a class of underactuated mechanical systems via interconnection and damping assignment," IEEE Transactions on Automatic Control, vol. 47, no. 8, pp. 1218-1233, 2002.

[11] B. Li, G. Xu, Y. Xia, W. Wang, and Z. Su, "Composite curve path following an underactuated AUV," Mathematical Problems in Engineering, vol. 2021, Article ID 6624893, 18 pages, 2021.

[12] V. I. Utkin, Sliding Modes in Control and Optimization, Springer, Berlin, Germany, pp. 1-11, 1992.

[13] B. Brogliato, A. Polyakov, and D. Efimov, "The implicit discretization of the supertwisting sliding-mode control algorithm," IEEE Transactions on Automatic Control, vol. 65, no. 8, pp. 3707-3713, 2020.

[14] S. Wang, J. Na, and Q. Chen, “Adaptive predefined performance sliding mode control of motor driving systems with disturbances," IEEE Transactions on Energy Conversion, vol. 36, no. 3, pp. 1931-1939, 2021.

[15] B. Lu, Y. Fang, and N. Sun, "Continuous sliding mode control strategy for a class of nonlinear underactuated systems," IEEE Transactions on Automatic Control, vol. 63, no. 10, pp. 3471-3478, 2018.

[16] J. Huang, S. Ri, T. Fukuda, and Y. Wang, "A disturbance observer based sliding mode control for a class of underactuated robotic system with mismatched uncertainties," IEEE Transactions on Automatic Control, vol. 64, no. 6, pp. 2480-2487, 2019.

[17] S. Wang, X. Wang, R. Xie, and C. Yao, "Robust backstepping control based on disturbance observer for hypersonic vehicle," Control and Decision, vol. 28, no. 10, pp. 1507-1512, 2013.

[18] O. Mofid, S. Mobayen, C. Zhang, and B. Esakki, "Desired tracking of delayed quadrotor UAV under model uncertainty and wind disturbance using adaptive super-twisting terminal sliding mode control," ISA Transactions, 2021.

[19] Z. Mao, X.-G. Yan, B. Jiang, and M. Chen, "Adaptive faulttolerant sliding-mode control for high-speed trains with actuator faults and uncertainties," IEEE Transactions on Intelligent Transportation Systems, vol. 21, no. 6, pp. 2449-2460, 2020.

[20] J. C. Lo and Y. H. Kuo, "Decoupled fuzzy sliding-mode control," IEEE Transactions on Fuzzy Systems, vol. 6, no. 3, pp. 426-435, 1998.

[21] H. Bayramoglu and H. Komurcugil, "Nonsingular decoupled terminal sliding-mode control for a class of fourth-order nonlinear systems," Communications in Nonlinear Science and Numerical Simulation, vol. 18, no. 9, pp. 2527-2539, 2013.

[22] S. Mobayen, S. Mostafavi, and A. Fekih, "Non-singular fast terminal sliding mode control with disturbance observer for 
underactuated robotic manipulators," IEEE Access, vol. 8, pp. 198067-198077, 2020.

[23] J. J. Slotine and S. S. Sastry, "Tracking control of non-linear systems using sliding surfaces, with application to robot manipulators $\dagger, "$ International Journal of Control, vol. 38, no. 2, pp. 465-492, 1983.

[24] H. Pan, G. Zhang, H. Ouyang, and L. Mei, "Novel fixed-time nonsingular fast terminal sliding mode control for secondorder uncertain systems based on adaptive disturbance observer," IEEE Access, vol. 8, pp. 126615-126627, 2020.

[25] A. Jouila and K. Nouri, "An adaptive robust nonsingular fast terminal sliding mode controller based on wavelet neural network for a 2-dof robotic arm," Journal of the Franklin Institute, vol. 357, no. 18, pp. 13259-13282, 2020.

[26] L. Liu, W. X. Zheng, and S. Ding, "High-order sliding mode controller design subject to lower-triangular nonlinearity and its application to robotic system," Journal of the Franklin Institute, vol. 357, no. 15, pp. 10367-10386, 2020.

[27] A. Guezmil, H. Berriri, R. Pusca, A. Sakly, R. Romary, and M. F. Mimouni, "High order sliding mode observer-based backstepping fault-tolerant control for induction motor," Asian Journal of Control, vol. 21, no. 1, pp. 33-42, 2019.

[28] H. Liu, J. Sun, J. Nie, and L. Zou, “Observer-based adaptive second-order non-singular fast terminal sliding mode controller for robotic manipulators," Asian Journal of Control, vol. 23, no. 4, pp. 1845-1854, 2020.

[29] Y. Wang, Y. Feng, X. Zhang, and J. Liang, "A new reaching law for antidisturbance sliding-mode control of PMSM speed regulation system," IEEE Transactions on Power Electronics, vol. 35, no. 4, pp. 4117-4126, 2020.

[30] A. Chalanga and F. Plestan, "High-order sliding-mode Control with predefined convergence time for electropneumatic actuator," IEEE Transactions on Control Systems Technology, vol. 29, no. 2, pp. 910-917, 2021.

[31] J. H. Hemandez, S. S. Cruz, R. Lopez-Gutierrez, A. GonzalezMendoza, and R. Lozanp, "Robust nonsingular fast terminal sliding-mode control for sit-to-stand task using a mobile lower limb exoskeleton," Control Engineering Practice, vol. 101, Article ID 104496, 2020.

[32] C. $\mathrm{Mu}$ and H. He, "Dynamic behavior of terminal sliding mode control," IEEE Transactions on Industrial Electronics, vol. 65, no. 4, pp. 3480-3490, 2018.

[33] M. Pu, T. Jiang, and K. Fu, "Finite time stable chattering-free reaching law design with bounded input," Control and Decision, vol. 33, no. 1, pp. 135-142, 2018.

[34] W. Gao, Theory and Design Method of Variable Structure Control, pp. 28-30, Science Press, Beijing, China, 1996.

[35] C. Wang, H. Xia, Y. Wang, and S. Ren, "Reduced-order disturbance observer-based adaptive reaching law control for discretized MIMO systems with unmatched uncertainties," Nonlinear Dynamics, vol. 105, no. 2, pp. 1611-1623, 2021.

[36] A. Bartoszewicz and P. Lesniewski, "New switching and nonswitching type reaching laws for SMC of discrete time systems," IEEE Transactions on Control Systems Technology, vol. 24, no. 2, pp. 670-677, 2016.

[37] H. Li and Y. Cai, "Sliding mode control with double power reaching law," Control and Decision, vol. 31, no. 3, pp. 498502, 2016.

[38] X. Meng, H. Yu, J. Zhang, T. Xu, H. Wu, and K. Yan, "Disturbance observer-based feedback linearization control for a quadruple-tank liquid level system," ISA Transactions, 2021. 